parameter (CAP) via liver ultrasound transient elastography. Mean values of Se intake and serum $\mathrm{Se}, \mathrm{tHg}$ and $\mathrm{MeHg}$ were calculated; univariate and multi-variate linear regressions were conducted between CAP and Se intake, serum Se, tHg and $\mathrm{MeHg}$, respectively with covariates of gender, age, ethnicity, tobacco smoke, BMI, educational level and average household income.

Results Mean \pm SD for Se intake was $101.9 \pm 70.8 \mathrm{mcg}$, serum Se $2.4 \pm 0.33 \mathrm{nmol} / \mathrm{L}$, serum tHg $5.6 \pm 11.4 \mathrm{nmol} / \mathrm{L}$, serum $\mathrm{MeHg} 4.6 \pm 9.8 \mathrm{nmol} / \mathrm{L}$, Se:tHg molar ratio of $1.1 \pm 0.8$, Se: $\mathrm{MeHg}$ molar ratio of $1.5 \pm 1.0$. The mean CAP was $257 \pm 66.2$ $\mathrm{dB} / \mathrm{m}$. Univariate linear regression showed a positive association between CAP and Se intake $(r=0.037, p<0.01)$, serum Se $(\mathrm{r}=19.7, p<0.001)$, Se:MeHg molar ratio $(\mathrm{r}=-4.9, p<0.001)$, Se:tHg molar ratio $(r=-2.8, p<0.001)$, respectively. After adjustment, associations between CAP and serum $\mathrm{MeHg}(\mathrm{r}=$ $0.14, p<0.05)$, serum Se $(\mathrm{r}=13.8, p<0.001)$ remained statistically significant, but the associations with the molar ratios no longer existed.

Conclusions Our results suggested that Se was associated with a decreased risk of NAFLD, whereas $\mathrm{tHg}$ and $\mathrm{MeHg}$ do not necessarily increase the disease risk. The molar ratios may present as early biomarkers for the prediction of NAFLD risk.

\section{IDDF2021-ABS-0156 SARCOPENIA ASSOCIATED WITH NON- ALCOHOL FATTY LIVER DISEASE (NAFLD) AND FIBROSIS AMONG ADULTS IN THE UNITED STATES: NATIONAL HEALTH AND NUTRITION EXAMINATION SURVEY (NHANES 2017-2018)}

${ }^{1}$ Siyu Dai*, ${ }^{2}$ Wentong Guo, 'Dingbo Shu, ${ }^{3}$ Feng Chen. 'School of Clinical Medicine, Hangzhou Normal University, Zhejiang Province, China; ${ }^{2}$ Department of Computer Science, The City University of Hong Kong, Hong Kong; ${ }^{3}$ Department of Paediatrics, Faculty of Medicine, The Chinese University of Hong Kong, Hong Kong

\subsection{6/gutjnl-2021-IDDF.97}

Background NAFLD and sarcopenia are common diseases affecting adult populations, constituting a major healthcare burden. The aim of this observational study is to explore associations between sarcopenia, NAFLD and stiffness, and to provide insights on prevention strategies targeted at these diseases.

Methods Subjects between 20-60 years were recruited from NHANES 2017-2018; those with alcohol abuse, underlying liver diseases and pregnancy were excluded. Dual energy Xray absorptiometry scan was applied for the measurement of appendicular lean mass (ALM). Sarcopenia was defined as a ratio of ALM/BMI below 0.789 for male, and 0.512 for female. NAFLD was determined using liver ultrasound transient elastography. Subjects with control attenuation parameter (CAP), which is an index for fatty liver severity, was categorized into no NAFLD $(\mathrm{CAP}<248 \mathrm{~dB} / \mathrm{m})$, mild $(248<\mathrm{CAP}<268 \mathrm{~dB} / \mathrm{m})$, moderate $(248<\mathrm{CAP}<268 \mathrm{~dB} / \mathrm{m})$, and severe $(>268 \mathrm{~dB} / \mathrm{m})$. Stiffness was examined simultaneously with cut-off values of $8.2,9.7$, and $13.6 \mathrm{Kpa}$. Student's t-test was performed to detect differences between groups; univariate and multivariate linear regression analysis were conducted to determine associations between sarcopenia, CAP and stiffness with adjustment of covariates (gender, age, ethnicity, tobacco use, BMI, educational level and average household income) when applicable.
Results A total of 3,510 subjects were included in the final analysis. Subjects with sarcopenia $(n=669)$; subjects with NAFLD (no:1584, mild: 257, moderate:187, severe:834); subject with stiffness (no: 2719, mild: 58, moderate: 47, severe: 38). For male with and without sarcopenia, mean CAP difference between was $5.33(p<0.001)$; mean stiffness difference was 1.89 ( $p=0.06)$. For female, mean CAP difference between was 6.19 ( $p<0.001)$; whereas no significance was found in stiffness. Linear regression showed that ALM/BMI and CAP were negatively associated in all subjects, and subjects with no and severe NAFLD (all $p$-values $<0.001$ ) before and after adjustment. No association was found for stiffness among study subjects.

Conclusions Our study demonstrated that adults with sarcopenia may have a higher risk of developing NAFLD and stiffness, indicating a new perspective on the early identification of high-risk populations.

\section{IDDF2021-ABS-0159 THE EFFECT OF CURCUMIN SUPPLEMENT AMONG OVERWEIGHT/OBESE NON- ALCOHOLIC FATTY LIVER DISEASE (NAFLD) PATIENTS}

Eduward Thendiono*. Internal Medicine Department, Bunda Hospital Gorontalo, Indonesia

\subsection{6/gutjn|-2021-IDDF.98}

Background Natural curcumin has been the subject of research for the treatment of Non-Alcoholic Fatty Liver Disease (NAFLD) due to antioxidant, anti-inflammatory, insulin-sensitizing, anti-steatotic, anti-fibrotic properties and affordable price. However, there are still scarce data about its benefit in a clinical setting.

Methods This is a retrospective study from the outpatient medical record, starting from 2nd January 2019 to 30th April 2020. Patients who diagnosed as fatty liver by ultrasonography (USG), body mass index (BMI) $>23 \mathrm{~kg} / \mathrm{m}^{2}$, received curcumin supplement daily for at least one month, non-alcoholic, elevated alanine aminotransferase (ALT), hypercholesterolemia, without comorbidities (such as diabetes mellitus, viral hepatitis, heart failure, nephrotic syndrome, infection case, kidney failure) were included for analysis. The primary outcomes are improvement of ALT level and regression of fatty liver by USG.

Results There were 48 patients who fulfilled the inclusion criteria. All of these patients were diagnosed as fatty liver (14 patients grade 1 and 34 patients grade 2 by USG) with elevated ALT (1 to 1,5 times upper normal limit), and hypercholesterolemia (non-High Density Lipoprotein C $>130 \mathrm{mg} / \mathrm{dL}$ ). All patients received curcumin supplement daily $(20 \mathrm{mg}$ of Curcumae xanthorrizae extract thrice a day), simvastatin $20 \mathrm{mg}$ daily and similar lifestyle modification advice. The longest treatment duration recorded was 6 months. Seventy-seven percent of patients (37/48) showed improvement in ALT level after 3 months of treatment $(\mathrm{p}<0.05)$. Eighty-seven percent of patients $(42 / 48)$ showed normal ALT levels after 6 months treatment $(\mathrm{p}<0.05)$. However, only twelve percent of patients $(6 / 48)$ showed improvement/regression of fatty liver in USG after 6 months of treatment $(p>0.05)$. There were no significant changes in BMI pre and post-treatment duration.

Conclusions Curcumin supplements may improving ALT level but have minimal effect on steatosis regression in overweight/ obese NAFLD patients. A randomized controlled clinical study is still needed to confirm the efficacy of curcumin in NAFLD. 